\title{
Acute caffeine administration affects zebrafish response to a robotic
}

\section{stimulus}

Fabrizio Ladu ${ }^{1}$, Violet Mwaffo ${ }^{1}$, Jasmine $\mathrm{Li}^{1}$, Simone Macrì ${ }^{1,2}$, Maurizio Porfiri ${ }^{1}$ *

${ }^{I}$ Department of Mechanical and Aerospace Engineering, New York University Polytechnic School of Engineering, Brooklyn 11201, New York, USA

${ }^{2}$ Section of Behavioural Neuroscience, Department of Cell Biology and Neuroscience, Istituto Superiore di Sanità, Roma 00161, Italy

*Corresponding author: mporfiri@nyu.edu, 718-260-3681 (phone), 718-260-3532 (fax)

\begin{abstract}
Zebrafish has been recently proposed as a valid animal model to investigate the fundamental mechanisms regulating emotional behavior and evaluate the modulatory effects exerted by psychoactive compounds. In this study, we propose a novel methodological framework based on robotics and information theory to investigate the behavioral response of zebrafish exposed to acute caffeine treatment. In a binary preference test, we studied the response of caffeine-treated zebrafish to a replica of a shoal of conspecifics moving in the tank. A purely data-driven information theoretic approach was used infer the influence of the replica on zebrafish behavior as a function of caffeine concentration. Our results demonstrate that acute caffeine administration modulates both the average speed and the interaction with the replica. Specifically, zebrafish exposed to elevated doses of caffeine show reduced locomotion and increased sensitivity to the motion of the replica. The methodology developed in this study may complement traditional experimental paradigms developed in the field of behavioral pharmacology.
\end{abstract}


Keywords: Anxiety, caffeine, fish replica, robotics, transfer entropy, zebrafish

\section{Introduction}

Anxiety-related disorders represent a widespread emotional disturbance affecting a large proportion of individuals [1]. Pharmacological studies on animal models may greatly aid our understanding of these disorders, by affording a tool upon which contributing variables can be identified, adjusted, and dissected. Among such models, zebrafish (Danio rerio) is becoming a species of choice [2-5], due to its short inter-generation time, fast reproduction rate, high stocking density, availability of genetic sequencing, complex social behavior, and elevated homology of structure and function with human nervous system [4, 6-11]. Among the different emotional domains addressed in zebrafish, anxiety-related behaviors have been analyzed in detail through different methodologies [12-19]. Beside the development of these methodologies, several authors also addressed the extent to which pharmacological treatments - known to modulate anxiety in humans and other mammals - affected zebrafish behavior [20].

Among different psychoactive compounds, acute caffeine treatment has been utilized to evoke anxiogenic behavioral responses in novel tank diving test [20-22] and light/dark box [23]. These studies have demonstrated that caffeine elicits characteristic diving behavior, increased thigmotaxis, reduced exploration, and increased erratic behavior in individual zebrafish. Such behavioral responses are, in turn, accompanied by elevated whole-body cortisol and brain c-fos $[20,21,24]$. While these efforts have contributed to the identification of the principal effects of caffeine on individual response, social behavior has yet to be fully examined $[12,25]$.

In this study, we propose the integration of robotics and information theory to investigate the effect of caffeine on zebrafish social behavior. To this aim, we addressed zebrafish response to a robotic stimulus resembling (in dimension and morphology) a shoal of four conspecifics. 
Individual behavior was studied under control conditions and in response to the acute administration of caffeine $(5,25$, and $50 \mathrm{mg} / \mathrm{L} ; 0.026,0.129$, and $0.257 \mathrm{mM}$ respectively). Specifically, in a binary preference test, we have studied the behavioral response of zebrafish subjects to a replica externally controlled using a mechanical platform [26, 27]. Based on experimental evidence indicating that anxiety-evoking stimuli result in increased shoaling tendency [12, 28-30], we expected that increasing caffeine concentration would result in a sronger interaction between the fish and the replica of conspecifics.

The interaction between the fish and the replica was quantified in an information theoretical framework through the transfer entropy, a construct often used to measure the mutual information flow between two dynamical systems and only recently proposed for the analysis of social behavior [26]. Transfer entropy quantifies the predictive power of a time series to anticipate another, possibly coupled, time series [31, 32]. Specifically, the transfer entropy from a dynamical system (e.g. the replica) to another (e.g. the fish) can be associated with a causal relationship between the two systems, manifested in the form of an uncertainty reduction computed directly from raw time series (e.g. the tracked motion of the replica and the fish in the tank) [32]. Differently from traditional measures of shoaling tendency based on physical proximity [12], our approach considers the swimming bouts that the live fish takes up from the prescribed motion of the replica. In other words, transfer entropy is used to approximate the degree of predictability of fish position in time from the prescribed motion of the replica. In addition to influencing the interaction with the replica, we also anticipated that acute caffeine administration would modulate fish speed and activity along the water column based on available literature $[12,20,25,33]$. 
The use of a robotic stimulus is grounded on the need to generate a controllable and repeatable visual stimulus $[19,34]$ on which to test the effect of caffeine. Specifically, the platform was pre-programmed independently of the motion of the live subjects to facilitate the assessment of information transfer. Given the unidirectional nature of the interaction, we treated the transfer entropy from the fish to the replica as a theoretical lower bound on which to contrast the transfer entropy from the replica to the fish.

\section{Materials and Methods}

This study was approved by the University Animal Welfare Committee of New York University under protocol number 13-1424.

\subsection{Animals}

A total of 40 wild-type zebrafish, approximately $3 \mathrm{~cm}$ in body length, were used in this experiment. All animals were purchased from an online aquarium source (LiveAquaria.com, Rhinelande, Wisconsin, USA) and were kept for a minimum of 12 days before experimentation. Fish were stocked at a housing density less than 1.06 fish/liter in 37.8 liter (10 gallons) tanks, in a room lit between 9 am to $11 \mathrm{pm}$ by full spectrum fluorescent light. Fish were fed around $7 \mathrm{pm}$ each day with commercial flake food (Hagen Corp./Nutrafin max, Mansfield, Massachusetts, USA) acquired from Petland Discount, Brooklyn, New York, USA. Temperature and acidity of the housing tanks were kept at $27 \pm 1^{\circ} \mathrm{C}$ and $7 \pm 1 \mathrm{pH}$, respectively.

\subsection{Platform and Replica}

The replica of the zebrafish shoal was designed in SolidWorks (Dassault Systèmes SolidWorks Corp., Waltham, Massachusetts, USA) and printed on a 3D prototyping machine (Stratasys, Dimension SST, Eden Prairie, Minnesota, USA) in rigid acrylonitrile butadiene styrene (ABS) 
material. Each replica was $3 \mathrm{~cm}$ in length and painted using waterproof spray paints (Krylon, Krylon Products Group, Cleveland, Ohio, USA) to imitate the color pattern of live zebrafish. To balance the visual cues offered to live fish, we also considered a control stimulus, comprised of the ABS skeleton holding the shoal replica to the end effector of the platform (see Figure 1).

The replica was actuated using an external platform (Figure 2 (a)). The platform design is similar to [26] and consisted of a robotic arm with three degrees of freedom, which enabled the rigid body motion of the fish shoal replica in $2 \mathrm{D}$. The platform comprised three rigid links, each actuated by a separate servo-motor (Futaba Corporation of America, Schaumburg, Illinois, USA; and Hitec RCD USA Inc., Poway, California, USA). The servo-motors were controlled using a pre-programmed microcontroller (Arduino Uno, Arduino, Italy), which allowed for imposing the desired motion to the end effector, where the replica was attached.

In our study, we selected a linear motion of $3 \mathrm{~cm} / \mathrm{s}$ superimposed to an oscillation with amplitude of $10^{\circ}$ at $2 \mathrm{~Hz}$ to imitate fish thrashing against the walls (based on personal observations of zebrafish tail beat undulations). As the replica traversed the tank, its orientation was reversed by $90^{\circ}$ every time the direction of motion was changed, so that the replica was always oriented at an average angle of $45^{\circ}$ with respect to the $x$-axis (Figure 2 (b)).

\subsection{Experimental Set-up}

The set-up consisted of a rectangular $76 \mathrm{~cm}$ x $30 \mathrm{~cm}$ x $30 \mathrm{~cm}$ tank (length, width, and height respectively). An overhead camera (Logitech Webcam Pro 9000, Newark, California, USA) was located $65 \mathrm{~cm}$ above the water surface to record the $2 \mathrm{D}$ motion of the subject and the end effectors at 15 frames per second. A second camera (Logitech Webcam Pro 9000, Newark, California, USA) was positioned in front of the tank to observe fish motion in the water column 
during habituation at 15 frames per second. The water depth was kept at $26 \mathrm{~cm}$ throughout all trials.

The tank was partitioned into three compartments of $10 \mathrm{~cm}, 56 \mathrm{~cm}$, and $10 \mathrm{~cm}$ length respectively using two transparent Plexiglas panels (see Figure 3); each $10 \mathrm{~cm}$ compartment constituted a stimulus compartment, while the central $56 \mathrm{~cm}$ compartment was used for the focal zebrafish. White contact paper was used to cover the bottom and all the lateral walls of the tank except of the one facing the frontal camera. The entire setup was isolated using black opaque curtains, to prevent external light exposure during the sessions. The only illumination was provided by two $25 \mathrm{~W}$ fluorescent tubes (All-Glass Aquarium, preheat aquarium lamp, UK) oriented along the tank.

Two platforms were placed on the opposite sides of the tank to move the replica and the control stimulus in the two stimulus compartments (see Figure 3).

\subsection{Drug Administration and Procedure}

Experiments were performed in August 2014. Caffeine $\left(\mathrm{C}_{8} \mathrm{H}_{10} \mathrm{~N}_{4} \mathrm{O}_{2}\right.$; product code C0750; CAS number 58-08-2) was purchased online from Sigma-Aldrich (St. Louis, Missouri, USA) in a powdered form, soluble in water. The study comprised four experimental conditions with 10 subjects each. The caffeine dose was varied across conditions, including $0 \mathrm{mg} / \mathrm{L}$ (control condition), $5 \mathrm{mg} / \mathrm{L}, 25 \mathrm{mg} / \mathrm{L}$, and $50 \mathrm{mg} / \mathrm{L}$. We originally planned to test the effects of a higher dose of caffeine $(100 \mathrm{mg} / \mathrm{L})$; however, a brief pilot experiment indicated that this dose had considerable side effects and may have significantly impaired animal welfare. For this reason, we removed this concentration from the study. Due to an experimental error detected after video examination, one of the trials for the $5 \mathrm{mg} / \mathrm{L}$ condition was discarded, leaving this condition with a total of nine subjects. 
Prior to experiments, fish were placed for 20 minutes in a beaker in which the appropriate caffeine concentration (including the control condition) was dissolved in water [20, 35]. After the 20-minute exposure period, fish were immediately transferred to the experimental tank using a hand net for a 20-minute recording session (10 minutes of habituation, 10 minutes of observation time). The same caffeine concentration was used in the experimental tank to maintain constant caffeine exposure throughout the trial. After the experiments, fish were placed in a different tank from the housing tank, to ensure that no animals would be used twice.

During habituation, two black plastic panels covered the stimulus compartments. In the first six minutes of the habituation time, the motors were kept off and we observed novelty effects in the focal subjects. Then, the motors were turned on and the black panels were removed manually. Trials were randomized, so that five trials of each condition were executed in the morning and the remaining five in the afternoon of a different day. All the experiments were performed between 9 am and $7 \mathrm{pm}$. The stimulus presentation was counterbalanced in each experimental condition, so that the shoal replica and the control stimulus were displayed for five trials on each side.

\subsection{Statistical Analysis}

All videos collected from the overhead camera were post-processed using a custom-made automatic tracking system [36] in MATLAB (R2014b; MathWorks, Natick, Massachusetts, USA), which allowed for tracking the 2D motion of focal subjects and end effectors. Trajectories were further manually verified and repaired using a MATLAB graphical user interface (GUI) by superposing the tracked trajectories to the video frames.

These data were used to compute fish average speed, estimate the preference index, and assess information transfer between the shoal replica and the fish [26]. The preference index was 
defined as the ratio between the time spent in the vicinity of the replica divided by the time spent in the vicinity of either the replica or the control stimulus [19]. Following [37], the fish was considered close to either the replica or the control stimulus if it were within $1 / 5$ of the middle compartment, corresponding to approximately four body lengths.

Transfer entropy, an information theoretic quantity often used to infer causal relationships between two dynamical systems [31], was here utilized to study the information transfer between fish and replica. If $X$ and $Y$ are two stationary Markov processes of order $k$ and $l$ respectively, the transfer entropy flow from $Y$ to $X$ is defined by

$$
\mathrm{T}_{Y \rightarrow X}=\sum_{x_{t+1}, x_{t}^{(k)}, y_{t}^{(l)}} p\left(x_{t+1}, x_{t}^{(k)}, y_{t}^{(l)}\right) \log _{2} \frac{p\left(x_{t+1} \mid x_{t}^{(k)}, y_{t}^{(l)}\right)}{p\left(x_{t+1} \mid x_{t}^{(k)}\right)}
$$

Here, $x_{t}^{(k)}$ and $y_{t}^{(l)}$ indicate the $k$ - and $l$-tuples comprising outcomes of $X$ and $Y$ from time $t-k+1$ and $t-l+1$, respectively, and the current time $t ; p\left(x_{t+1}, x_{t}^{(k)}, y_{t}^{(l)}\right)$ is the joint probability distribution between the value of the process $X$ at time $t+1$ and both the histories of $X$ and $Y$; $p\left(x_{t+1} \mid x_{t}^{(k)}, y_{t}^{(l)}\right)$ is the conditional probability distribution of $X$ at time $t+1$ given both the histories of $X$ and $Y$; and $p\left(x_{t+1} \mid x_{t}^{(k)}\right)$ is the conditional probability distribution of $X$ at time $t+1$ given only the time history of $X$; $\square$ denotes summation over all possible outcomes. This quantity measures the reduction in uncertainty in $X$ at time $t+1$ given both the time histories of $X$ and $Y$ in comparison of given only the time history of $X$; for example, if $X$ is independent of $Y$ then $\mathrm{T}_{Y \rightarrow X}=0$. The transfer entropy is not symmetric, so that the information flow from $Y$ to $X$ is generally different than the information flow from $X$ to $Y$. In this sense, given the direction of dominant information flow, a cause-effect relationship may be inferred between two dynamical systems [32].

The transfer entropy analysis was conducted with respect to the motion of the fish and the 
replica about the short side of the tank, along which the replica was moved. Each time series comprised 9000 samples. Transfer entropy was computed in both directions (fish to replica and replica to fish) and for all conditions. Transfer entropy from the replica to the fish should be related to the reduction in the uncertainty of fish behavior due to the knowledge of the replica motion. The more fish behavior is influenced by the replica, the higher would be the transfer entropy from the replica to the fish. This quantity should be contrasted with the transfer entropy from the fish to the replica. It is the asymmetry between these two quantities, transfer entropy from fish to replica and transfer entropy from replica to fish, that defines the direction of the information flow.

Computations were carried out using Process_Network_v1.4 software [38] running in MATLAB. The software parameters were set to the default values except for the number of bins to compute the empirical probability distribution and the number of workers for parallel processing. The number of bins was chosen to be 10 , so that the length of each bin is approximately one zebrafish body length. Data was not downsampled and a first-order Markov chain was considered in the computation so that $k=l=1$. The number of parallel workers was set to four, corresponding to the capacity of our computing system, resulting into a computational time of approximately three minutes for each trial. The number of shuffled surrogate tests was set to 100 and no transformation or data filtering were allowed. Computations were verified by downsampling the data and calculating a variant of Equation (1) that includes a lag between the two time series [39].

Videos acquired by the front camera were processed using Observer 2.0 (Noldus, Wageningen, the Netherlands), to score zebrafish diving along the water column. For this purpose, we divided the water column into two parts (top half and bottom half) to study novelty 
effects in the first six minutes of habituation. We computed the time spent in the top half and the number of transitions between the two halves.

Statistical analysis was performed using repeated measures ANOVA for split-plot designs, with conditions ( $0 \mathrm{mg} / \mathrm{L}, 5 \mathrm{mg} / \mathrm{L}, 25 \mathrm{mg} / \mathrm{L}$, and $50 \mathrm{mg} / \mathrm{L})$ as between-subjects factors, and 10 one-minute time intervals (for average speed) and direction of the information flow (for transfer entropy). Furthermore, in comparison between both directions of the information flow inside each condition we performed one-way ANOVA. One-way ANOVA was also used to study differences in the preference index, time spent in the top half, and number of transitions between the top and bottom halves between conditions. To test whether during habituation fish preferred the bottom half of the test tank, we performed a one-sample two-tail t-test. Post-hoc tests for the analysis of average speed, preference index, time spent in the top half, and number of transitions between the top and bottom halves were performed using Tukey's HSD. Significance was set at $\mathrm{p}<0.05$.

\section{Results}

Figure 4 demonstrates that the average speed of the focal subjects decreases as a function of caffeine administration $\left(\mathrm{F}_{3,35}=5.825, \mathrm{p}<0.05\right)$. Post-hoc comparisons indicate that $25 \mathrm{mg} / \mathrm{L}$ - and 50mg/L-treated subjects swam at a lower speed compared to control subjects $(\mathrm{p}<0.05)$. Predictably, experimental subjects displayed a significant preference for the bottom half of the tank during habituation $\left(\mathrm{t}_{1,38}=3.30, \mathrm{p}<0.01\right)$, but caffeine administration did not result in variations of such preference (Figure 5(a)). However, Figure 5(b) shows that caffeine administration modulated the number of transitions between the top and bottom halves of the $\operatorname{tank}\left(\mathrm{F}_{3,35}=4.656, \mathrm{p}<0.01\right)$. Post-hoc comparisons indicate that fish treated with the highest 
concentration transitioned less frequently between the two halves of the tank than subjects treated with low and intermediate doses $(\mathrm{p}<0.05)$.

Figure 6 indicates that conditions are indistinguishable in terms of information flow between fish and replica $\left(\mathrm{F}_{3,35}=0.714, \mathrm{p}=0.5501\right)$. However, one-way ANOVA to compare the direction of the information flow demonstrates that at high caffeine concentration, the transfer entropy from the fish to the replica is less than the transfer entropy from the replica to the fish $\left(\mathrm{F}_{1,18}=10.377, \mathrm{p}<0.01\right.$ for $25 \mathrm{mg} / \mathrm{L}$ and $\mathrm{F}_{1,18}=6.041, \mathrm{p}<0.05$ for $\left.50 \mathrm{mg} / \mathrm{L}\right)$. The significance of these comparisons is unaltered by varying the time lag between the time series within five frames. With respect to low caffeine concentration, the difference between the transfer entropy from the fish to the replica and the transfer entropy from the replica to the fish failed to reach statistical significance $\left(\mathrm{F}_{1,18}=4.398, \mathrm{p}=0.0522\right.$ for $\left.5 \mathrm{mg} / \mathrm{L}\right)$. Downsampling the data with a sampling period between 1 and $4 \mathrm{~s}$ does not induce any inversion of the measured direction of information flow for all conditions.

Finally, Figure 7 indicates that the preference index does not vary with caffeine concentration $\left(\mathrm{F}_{3,35}=0.666, \mathrm{p}=0.578\right)$, with live fish spending an approximately equal amount of time in the vicinity of the replica and the control stimulus. Fish spent on average a half of the time in the central portion of the middle compartment (3/5 of the total length), away from the two stimuli (0 mg/L: $64.21 \pm 17.38 \%, 5 \mathrm{mg} / \mathrm{L}: 48.22 \pm 20.96 \%, 25 \mathrm{mg} / \mathrm{L}: 48.39 \pm 22.02 \%$, and $50 \mathrm{mg} / \mathrm{L}: 45.14 \pm 27.60 \%$ ).

\section{Discussion}

In this work, we have studied the behavioral response of caffeine-treated zebrafish presented with the visual stimulus of a shoal of conspecifics through an external mechanical platform. Our results suggest that in addition to altering absolute levels of locomotion, caffeine administration 
modulated the interaction between experimental subjects and the replica measured in an information theoretic context. Conversely, in partial contrast with our original predictions, caffeine administration did not apparently modify the preference index in fish-replica interactions.

Specifically, we have found that zebrafish average speed in response to intermediate and high caffeine concentrations was significantly lower than the control group. The interaction between live fish and the replica was studied through the concept of transfer entropy to quantify causal relationships between the motions of the fish and the pre-programmed replica. Control and $5 \mathrm{mg} / \mathrm{L}$ individuals did not display a robust interaction with the replica, whereby the difference between the transfer entropies from fish to replica and replica to fish failed to reach statistical significance. On the other hand, the behavior of subjects treated with intermediate and high caffeine concentrations was influenced by the motion of the replica, with the transfer entropy from fish to replica being less than transfer entropy from replica to fish.

The observed variation in fish activity as a function of caffeine administration is in accordance with previous findings, which have shown that zebrafish speed decreases as caffeine concentration increases $[33,35]$. This effect can be associated with the capacity of caffeine to inhibit the adenosine $A_{1}$ and $A_{2 A}$ receptors $[33,40]$. Specifically, the reduction in the average speed at intermediate and high caffeine doses could be attributed to the blockade of the $A_{1}$ receptor, while the blockade of $\mathrm{A}_{2 \mathrm{~A}}$ receptor could be related to the increase in the average speed at the lowest doses. Consistent with the literature on novelty tank diving tests [20, 21, 25], we found that fish exhibited a preference for the bottom half of the test tank during habituation. However, due the inter-individual variability, the limited number of subjects, and the more 
modest caffeine treatment compared to the literature, we did not observe major effects associated with caffeine treatment.

The variation of the fish speed may be further amplified by social behavior, whereby fish could try to match their speed with the replica $(3 \mathrm{~cm} / \mathrm{s})$ when exposed to higher caffeine doses. This hypothesis rests on the anxiogenic action of caffeine and experimental evidence indicating that anxiety could enhance shoaling tendency [28, 30, 41]. Thus, it is tenable to hypothesize that intermediate and high caffeine concentrations evoke anxiety-related behavioral responses, which, in turn, result into a more robust shoaling tendency. Transfer entropy data seem to further support this possibility, whereby only fish treated at intermediate and high caffeine concentrations were robustly influenced by the motion of the replica.

Although the replica was designed to mimic a shoal of conspecifics in their morphophysiology and swimming pattern, it is unlikely that fish perceive the replica as a live shoal. In fact, untreated fish exhibit a rather modest preference for the replica in comparison with related experimental studies using live fish as stimuli [37], where preference indices ranged between 70 and $80 \%$ and the time spent in the center of the middle compartment was only $20 \%$ of the total experiment time for live stimuli comprising one or ten conspecifics. While not perceived as a shoal of conspecifics, the presence of the replica may enrich the environment in a social context [42-45], which resonates with the anxiogenic action of caffeine to influence fish behavior. For example, the replica could constitute a stimulus analogous to computer animations, which, in turn, have been proposed as an effective tool to proxy social context [43].

The information flow between the shoal replica and the fish was quantified using the theoretic construct of transfer entropy, which we have recently proposed as a valid tool to study social interactions [26]. Although the numerical estimation of transfer entropy may be 
technically challenging [39], information flow measured in this sense highlights nonlinear relationships over large interaction delays, which may be misinterpreted using correlation-based methods [46]. Through the use of transfer entropy, we introduce an objective measure of social influence that is entirely data-driven and prescinds from a-priori assumptions on the cues underpinning social behavior. Specifically, the approach only requires the time traces of the individuals' motion to elucidate information flow between them, in the form of swimming bouts that are initiated by an individual and then taken up by the other. Simulated experiments of collective behavior [47] suggest that such an information theoretical approach could contribute to the study of social interactions in fish shoals, where information transfer is not limited to pairwise relationships.

The mechanical platform is another important element of this study, which enables the delivery of a repeatable and controllable visual stimulus to live subjects [19, 34]. Our prior work on the use of robotic stimuli has demonstrated that inter-individual response to a robotic stimulus is more consistent than that exhibited in response to live conspecifics and allopatric predators, thereby increasing experimental statistical power [19, 34]. Leveraging robotics, we have designed a novel experimental protocol to investigate the psychoactive action of caffeine on zebrafish social behavior; we believe that this aspects constitutes a source of novelty compared to the majority of available literature $[12,25]$, which has predominantly focused on individual response.

Overall, this study offers a first demonstration of the possibility of using robotics and information theory to investigate the effect of anxiogenic compounds on zebrafish social behavior. Just as robotics enable tailorable and consistent visual stimuli creating a social context, information theory affords the quantification of the influence of the stimulus on zebrafish 
behavior. While we focused on acute caffeine treatment, the same methodology can be applied to the analysis of other psychoactive substances [28, 48-50] eliciting anxiogenic or anxiolytic responses, as well as developmental and chronic treatments [51, 52].

\section{Acknowledgements}

The authors gratefully acknowledge Paul Phamduy and Sukhgewanpreet Singh for their help in developing the mechanical platforms and Tiziana Bartolini, Sachit Butail, and Jeffrey Laut for their careful review of the manuscript. This work was supported by the National Science Foundation under grant nos. CMMI-0745753, CMMI-1129820, CMMI-1433670, and DGE0741714.

\section{References}

[1] Kessler RC, Sampson NA, Berglund P, Gruber MJ, Al-Hamzawi A, Andrade L, et al. Anxious and non-anxious major depressive disorder in the World Health Organization World Mental Health Surveys. Epidemiology and Psychiatric Sciences. 2015;FirstView:1-17.

[2] Kalueff AV, Stewart AM, Gerlai R. Zebrafish as an emerging model for studying complex brain disorders. Trends in Pharmacological Sciences. 2014;35:63-75.

[3] Lieschke GJ, Currie PD. Animal models of human disease: zebrafish swim into view. Nature Reviews Genetics. 2007;8:353-67.

[4] Miklósi A, Andrew RJ. The zebrafish as a model for behavioral studies. Zebrafish. 2006;3:227-34.

[5] Gerlai R. Using zebrafish to unravel the genetics of complex brain disorders. Current Topics in Behavioral Neurosciences. 2012;12:3-24. 
[6] Postlethwait JH, Woods IG, Ngo-Hazelett P, Yan YL, Kelly PD, Chu F, et al. Zebrafish comparative genomics and the origins of vertebrate chromosomes. Genome Research. 2000;10:1890-902.

[7] Panula P, Chen YC, Priyadarshini M, Kudo H, Semenova S, Sundvik M, et al. The comparative neuroanatomy and neurochemistry of zebrafish CNS systems of relevance to human neuropsychiatric diseases. Neurobiology of Disease. 2010;40:46-57.

[8] Buske C, Gerlai R. Shoaling develops with age in Zebrafish (Danio rerio). Progress in Neuro-Psychopharmacology and Biological Psychiatry. 2011;35:1409-15.

[9] Miller N, Gerlai R. From Schooling to Shoaling: Patterns of Collective Motion in Zebrafish (Danio rerio). PLoS ONE. 2012;7:e48865.

[10] Miller NY, Gerlai R. Shoaling in zebrafish: what we don’t know. Reviews in the Neurosciences. 2011;22:17-25.

[11] Lawrence C. The husbandry of zebrafish (Danio rerio): A review. Aquaculture. 2007;269:120.

[12] Maximino C, de Brito TM, da Silva Batista AW, Herculano AM, Morato S, Gouveia A, Jr. Measuring anxiety in zebrafish: a critical review. Behavioural Brain Research. 2010;214:157-71. [13] Maximino C, de Brito TM, Colmanetti R, Pontes AA, de Castro HM, de Lacerda RI, et al. Parametric analyses of anxiety in zebrafish scototaxis. Behavioural Brain Research. 2010;210:17.

[14] Maximino C, de Brito TM, Dias CA, Gouveia A, Jr., Morato S. Scototaxis as anxiety-like behavior in fish. Nature Protocols. 2010;5:209-16.

[15] Serra E, Medalha C, Mattioli R. Natural preference of zebrafish (Danio rerio) for a dark environment. Brazilian Journal of Medical and Biological Research. 1999;32:1551-3. 
[16] Blaser RE, Rosemberg DB. Measures of anxiety in zebrafish (Danio rerio): dissociation of black/white preference and novel tank test. PLoS ONE. 2012;7:e36931.

[17] Barba-Escobedo PA, Gould GG. Visual social preferences of lone zebrafish in a novel environment: strain and anxiolytic effects. Genes, Brain and Behavior. 2012;11:366-73.

[18] Qin M, Wong A, Seguin D, Gerlai R. Induction of social behavior in zebrafish: live versus computer animated fish as stimuli. Zebrafish. 2014;11:185-97.

[19] Ladu F, Bartolini T, Panitz SG, Butail S, Macri S, Porfiri M. Live predators, robots, and computer-animated images elicit differential avoidance responses in zebrafish. Zebrafish. [20] Cachat JM, Stewart A, Grossman L, Gaikwad S, Kadri F, Chung KM, et al. Measuring behavioral and endocrine responses to novelty stress in adult zebrafish. Nature Protocols. 2010;5:1786-99.

[21] Egan RJ, Bergner CL, Hart PC, Cachat JM, Canavello PR, Elegante MF, et al. Understanding behavioral and physiological phenotypes of stress and anxiety in zebrafish. Behavioural Brain Research. 2009;205:38-44.

[22] Wong K, Elegante MF, Bartels BK, Elkhayat SI, Tien DH, Roy S, et al. Analyzing habituation responses to novelty in zebrafish (Danio rerio). Behavioural Brain Research. 2010;208:450-7.

[23] Maximino C, da Silva AW, Gouveia A, Jr., Herculano AM. Pharmacological analysis of zebrafish (Danio rerio) scototaxis. Progress in Neuro-Psychopharmacology and Biological Psychiatry. 2011;35:624-31.

[24] Cachat JM, Canavello PR, Elegante MF, Bartels BK, Elkhayat SI, Hart PC, et al. Modeling stress and anxiety in zebrafish. In: Kalueff AV, editor. Zebrafish models in neurobehavioral research: Springer; 2011. p. 73-88. 
[25] Stewart A, Wu N, Cachat J, Hart P, Gaikwad S, Wong K, et al. Pharmacological modulation of anxiety-like phenotypes in adult zebrafish behavioral models. Progress in NeuroPsychopharmacology and Biological Psychiatry. 2011;35:1421-31.

[26] Butail S, Ladu F, Spinello D, Porfiri M. Information flow in animal-robot interactions. Entropy. 2014;16:1315-30.

[27] Phamduy P, Polverino G, Fuller RC, Porfiri M. Fish and robot dancing together: bluefin killifish females respond differently to the courtship of a robot with varying color morphs. Bioinspiration \& Biomimetics. 2014;9:036021.

[28] Reyhanian N, Volkova K, Hallgren S, Bollner T, Olsson PE, Olsen H, et al. 17alpha-Ethinyl estradiol affects anxiety and shoaling behavior in adult male zebra fish (Danio rerio). Aquatic Toxicology. 2011;105:41-8.

[29] Speedie N, Gerlai R. Alarm substance induced behavioral responses in zebrafish (Danio rerio). Behavioural Brain Research. 2008;188:168-77.

[30] Grossman L, Utterback E, Stewart A, Gaikwad S, Chung KM, Suciu C, et al. Characterization of behavioral and endocrine effects of LSD on zebrafish. Behavioural Brain Research. 2010;214:277-84.

[31] Schreiber T. Measuring information transfer. Physical Review Letters. 2000;85:461-4. [32] Hlavackova-Schindler K, Palus M, Vejmelka M, Bhattacharya J. Causality detection based on information-theoretic approaches in time series analysis. Physics Reports. 2007;1:1-46. [33] Gupta P, Khobragade S, Shingatgeri V, Rajaram S. Assessment of locomotion behavior in adult zebrafish after acute exposure to different pharmacological reference compounds. Drug Development and Therapeutics. 2014;5:127-33. 
[34] Spinello C, Macri S, Porfiri M. Acute ethanol administration affects zebrafish preference for a biologically inspired robot. Alcohol. 2013;47:391-8.

[35] Wong K, Stewart A, Gilder T, Wu N, Frank K, Gaikwad S, et al. Modeling seizure-related behavioral and endocrine phenotypes in adult zebrafish. Behavioral Brain Research. 2010;1348:209-15.

[36] Butail S, Bartolini T, Porfiri M. Collective response of zebrafish shoals to a free-swimming robotic fish. PLoS ONE. 2013;8:e76123.

[37] Polverino G, Abaid N, Kopman V, Macri S, Porfiri M. Zebrafish response to robotic fish: preference experiments on isolated individuals and small shoals. Bioinspiration \& Biomimetics. 2012;7:036019.

[38] Ruddell BL, Oberg N, Garcia M, Kumar P. Using information-theoretic statistics in MATLAB to understand how ecosystems affect regional climates. MATLAB Digest Academic Edition. 2010. p. www.mathworks.com/academia.

[39] Ruddell BL, Kumar P. Ecohydrologic process networks: 1. Identification. Water Resources Research. 2009;45:W03419.

[40] Yacoubi ME, Ledent C, Ménard JF, Parmentier M, Costentin J, Vaugeois JM. The stimulant effects of caffeine on locomotor behaviour in mice are mediated through its blockade of adenosine A2A receptors. British Journal of Pharmacology. 2000;129:1465-73.

[41] Pham M, Raymond J, Hester J, Kyzar E, Gaikwad S, Bruce I, et al. Assessing social behavior phenotypes in adult zebrafish: shoaling, social preference, and mirror biting tests. Zebrafish protocols for neurobehavioral research: Springer; 2012. p. 231-46.

[42] Pritchard VL, Lawrence J, Butlin RK, Krause J. Shoal choice in zebrafish, Danio rerio: the influence of shoal size and activity. Animal Behaviour. 2001;62:1085-8. 
[43] Saverino C, Gerlai R. The social zebrafish: behavioral responses to conspecific, heterospecific, and computer animated fish. Behavioural Brain Research. 2008;191:77-87.

[44] Spence R, Gerlach G, Lawrence C, Smith C. The behaviour and ecology of the zebrafish, Danio rerio. Biological Reviews of the Cambridge Philosophical Society. 2008;83:13-34.

[45] Butail S, Polverino G, Phamduy P, Del Sette F, Porfiri M. Influence of robotic shoal size, configuration, and activity on zebrafish behavior in a free-swimming environment. Behavioural Brain Research. 2014;275:269-80.

[46] Li W. Mutual information functions versus correlation functions. Journal of Statistical Physics. 1990;60:823-37.

[47] Wang XR, Miller JM, Lizier JT, Prokopenko M, Rossi LF. Quantifying and tracing information cascades in swarms. PLoS ONE. 2012;7:e40084.

[48] Oliveira TA, Koakoski G, Kreutz LC, Ferreira D, da Rosa JG, de Abreu MS, et al. Alcohol impairs predation risk response and communication in zebrafish. PLoS ONE. 2013;8:e75780. [49] Miller N, Greene K, Dydinski A, Gerlai R. Effects of nicotine and alcohol on zebrafish (Danio rerio) shoaling. Behavioural Brain Research. 2013;240:192-6.

[50] Ladu F, Butail S, Macri S, Porfiri M. Sociality modulates the effects of ethanol in zebrafish. Alcoholism: Clinical and Experimental Research. 2014;38:2096-104.

[51] Guo S. Using zebrafish to assess the impact of drugs on neural development and function. Expert Opinion on Drug Discovery 2009;4:715-26.

[52] Gerlai R, Lee V, Blaser R. Effects of acute and chronic ethanol exposure on the behavior of adult zebrafish (Danio rerio). Pharmacology Biochemistry and Behavior. 2006;85:752-61. 


\section{Figure captions}

Figure 1: Visual stimuli. On the left, the shoal of four zebrafish, and on the right the control stimulus.

Figure 2: Illustration of the platform (a) and top view depicting the linear motion of the end effector (b).

Figure 3: Illustration of the experimental set-up, consisting of a tri-partitioned tank with two identical platforms moving a shoal replica and a control stimulus.

Figure 4: Average speed of focal subjects for different caffeine levels. Curly braces identify conditions significantly different in post-hoc analysis. Error bars represent \pm standard errors.

Figure 5: Diving test during the first six minutes of habituation. Time spent by focal fish in the top half of the water column is displayed for each condition (a), and number of transitions between the top and bottom half (b). Curly braces identify conditions significantly different in post-hoc analysis. Error bars represent \pm standard errors.

Figure 6: Information flow between the replica and the fish, measured through transfer entropy. Transfer entropies from fish to replica (fish $\rightarrow$ replica) and vice versa (replica $\rightarrow$ fish) are displayed for each condition. Curly braces identify conditions significantly different in one-way ANOVA. Error bars represent \pm standard errors.

Figure 7: Spatial preference toward the replica. Error bars represent \pm standard errors. 
Figure(s)
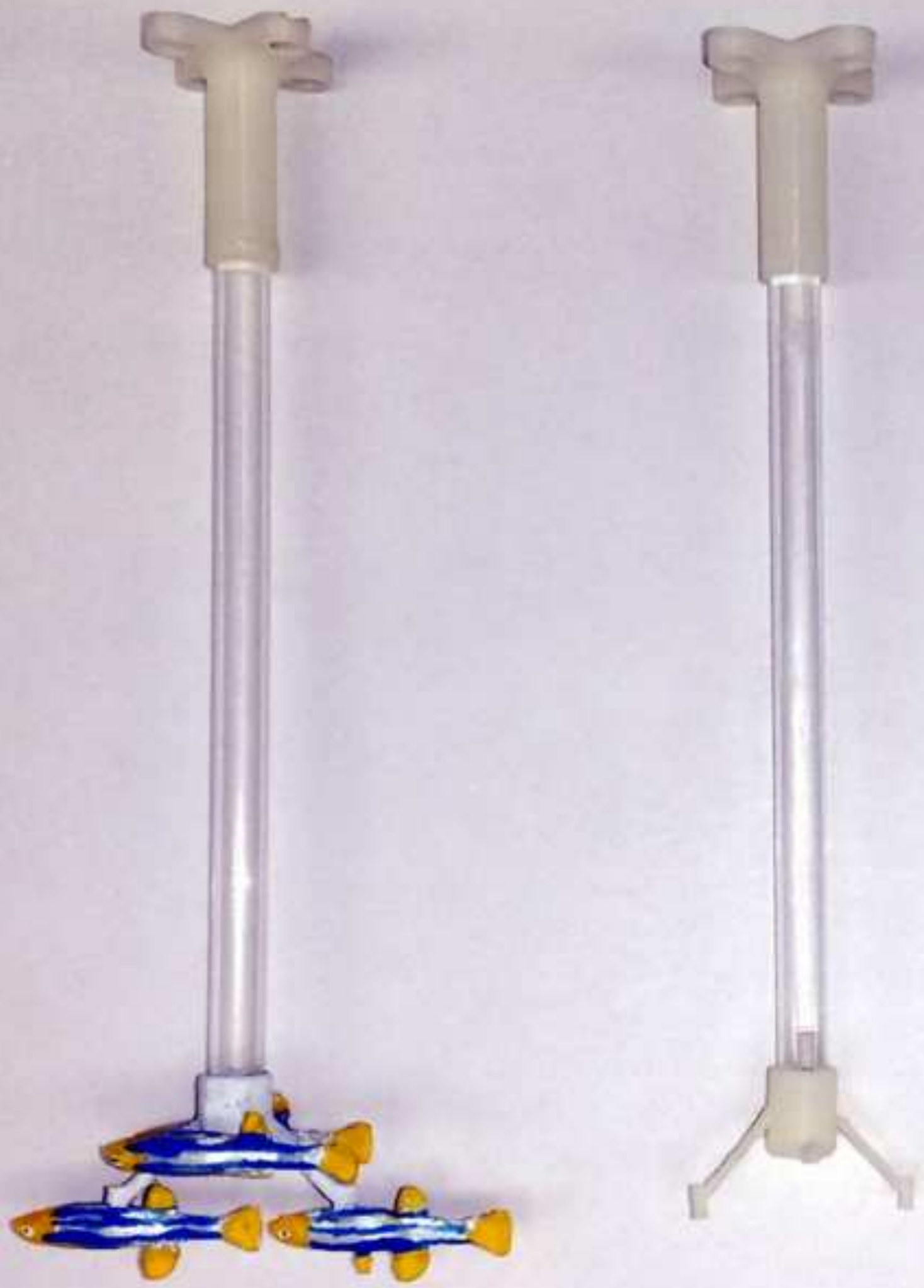


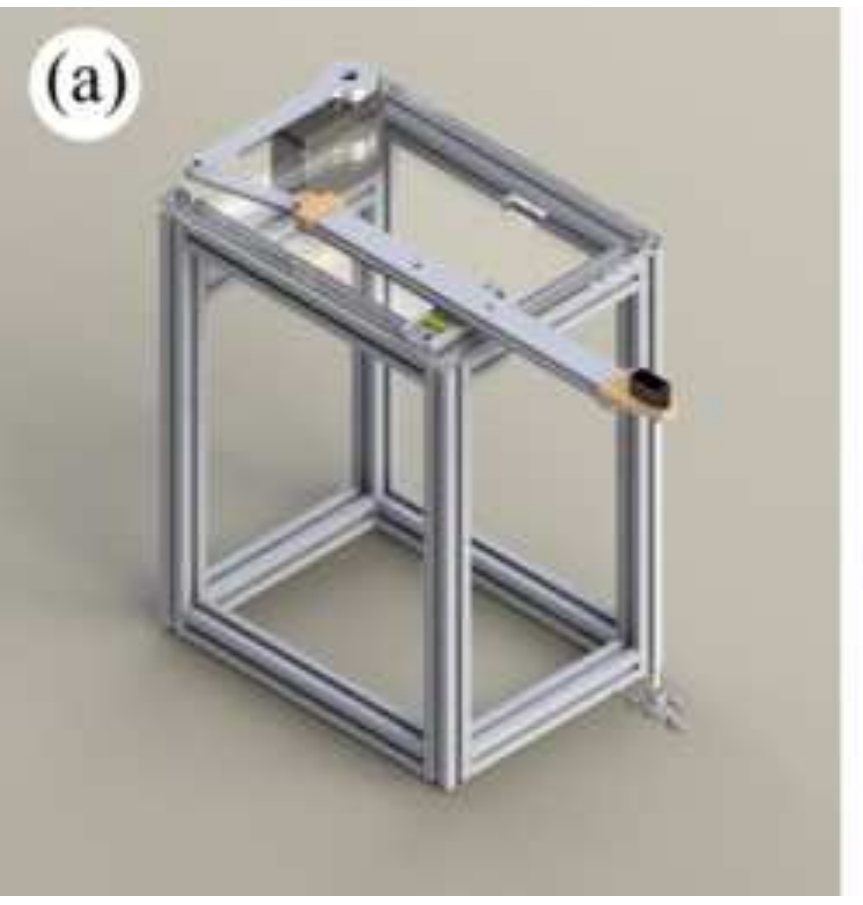

\section{(b)}

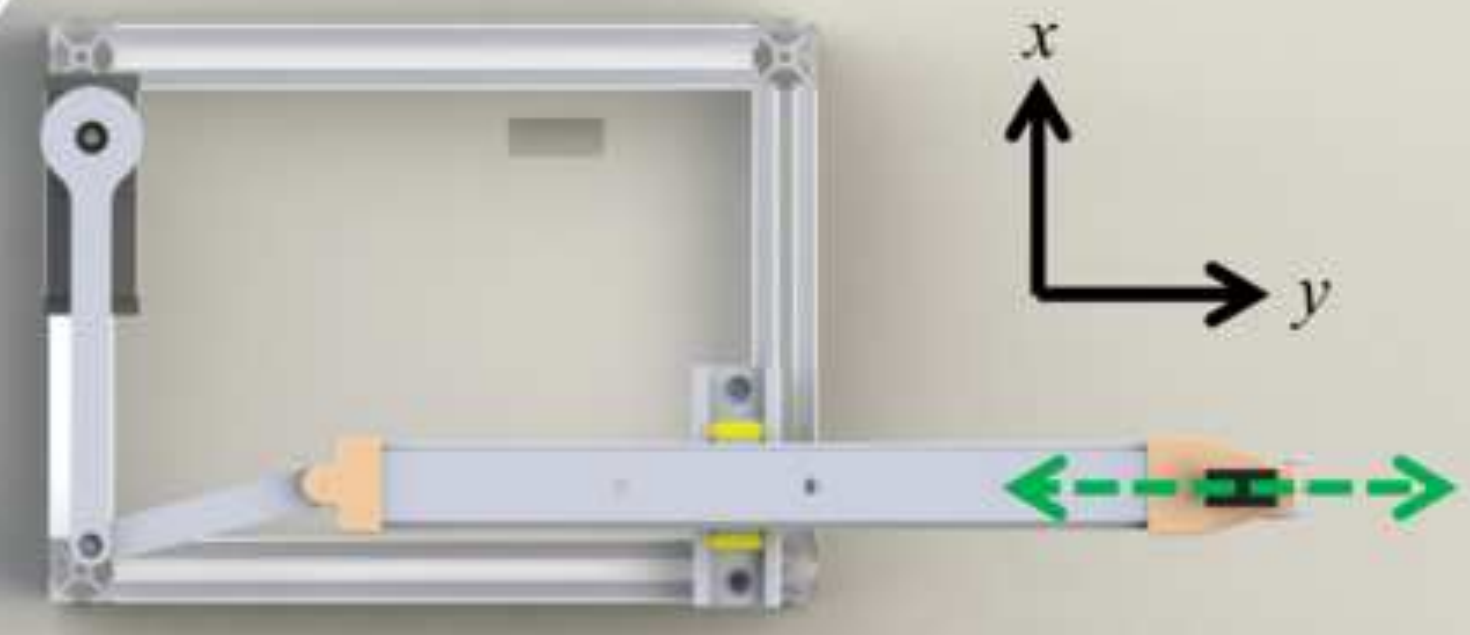




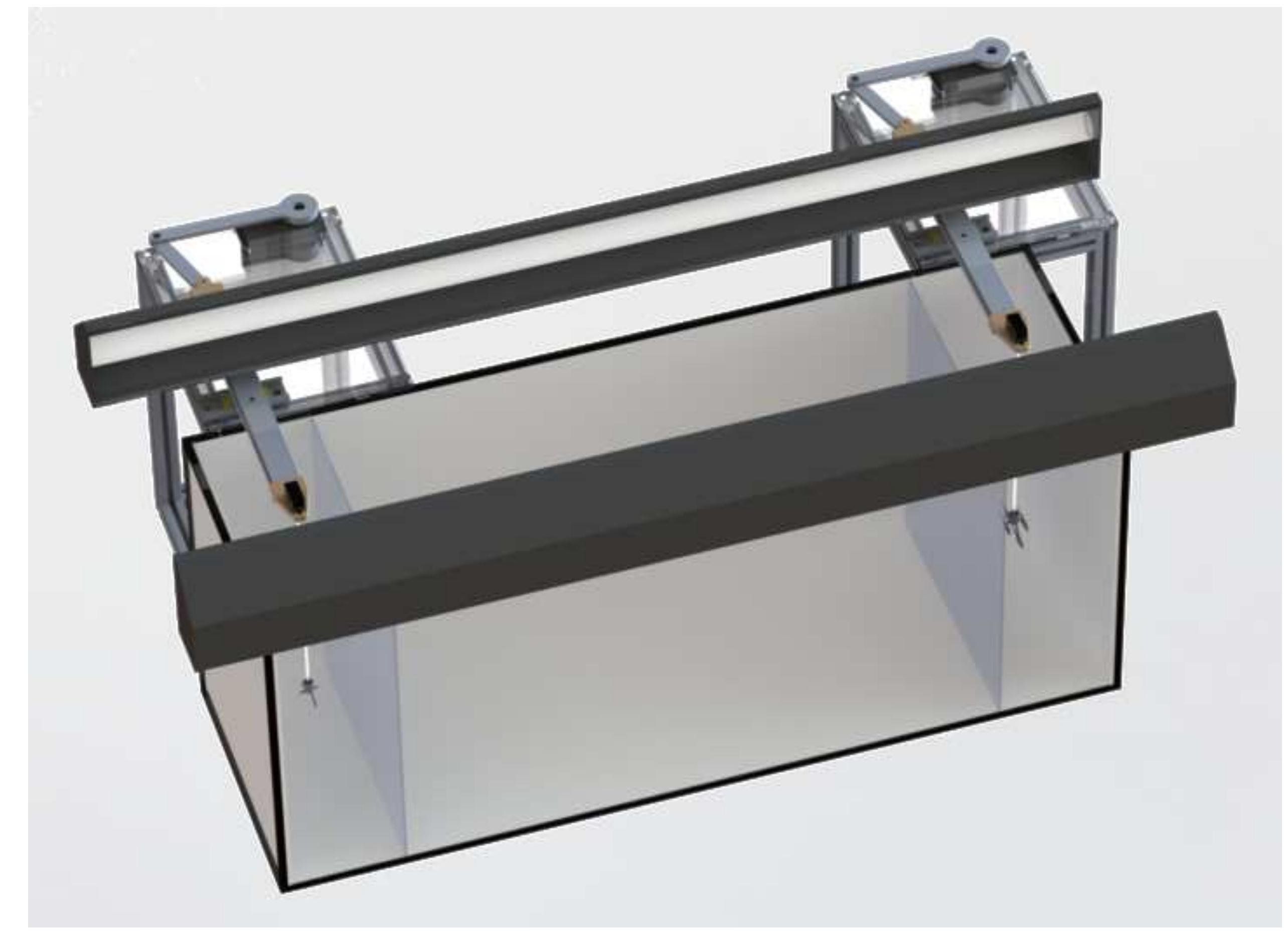

(s)




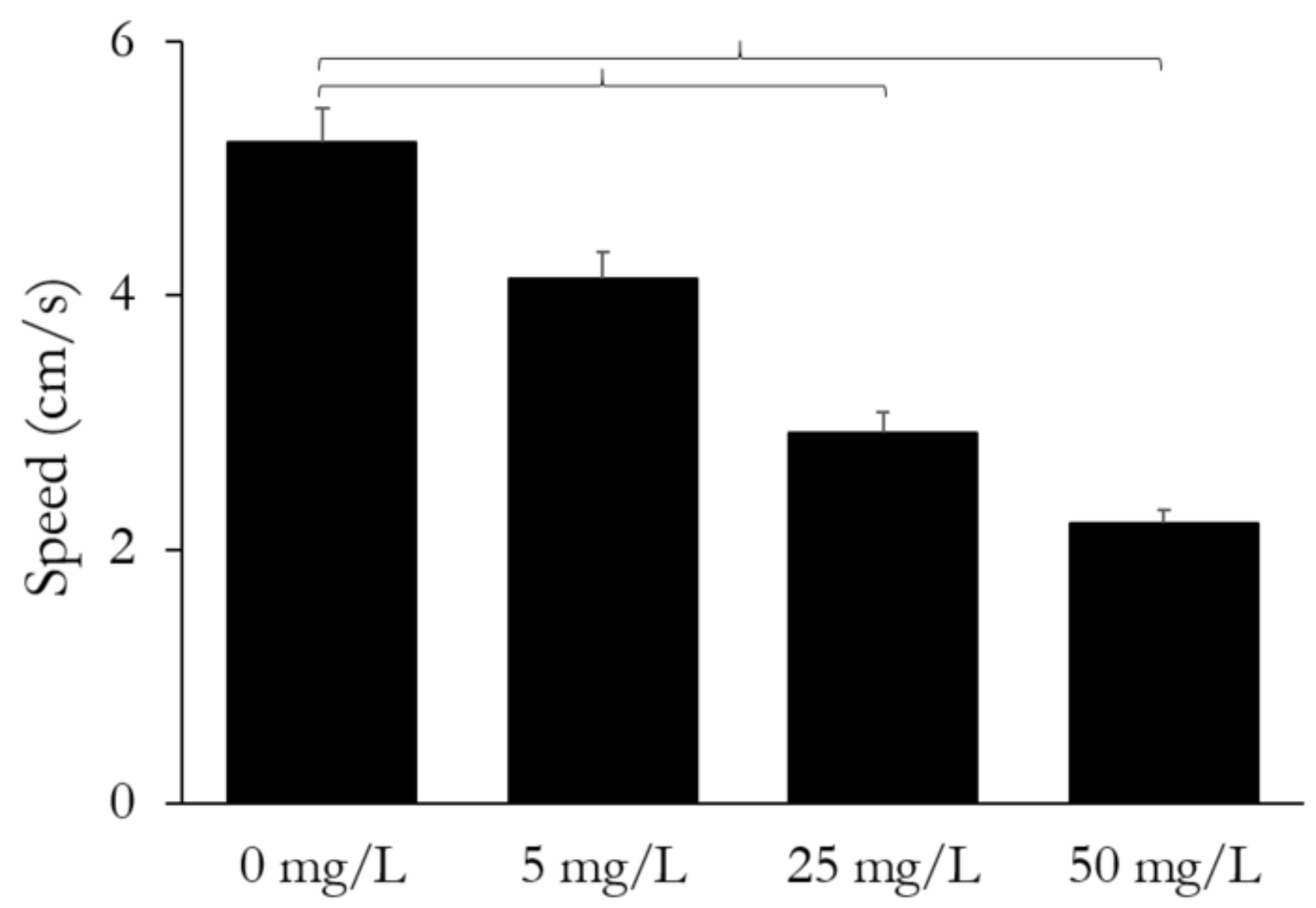


(a)

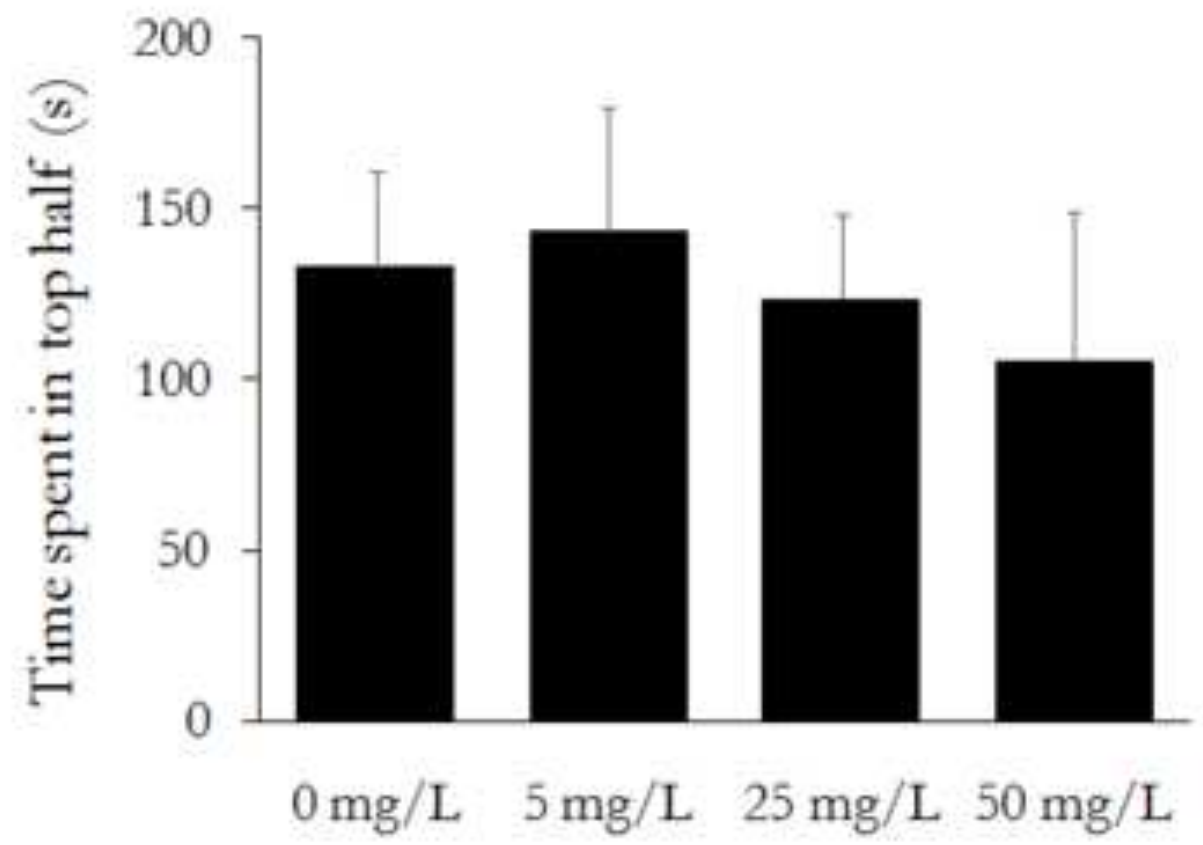

$0 \mathrm{mg} / \mathrm{L} \quad 5 \mathrm{mg} / \mathrm{L} \quad 25 \mathrm{mg} / \mathrm{L} \quad 50 \mathrm{mg} / \mathrm{L}$ (b)

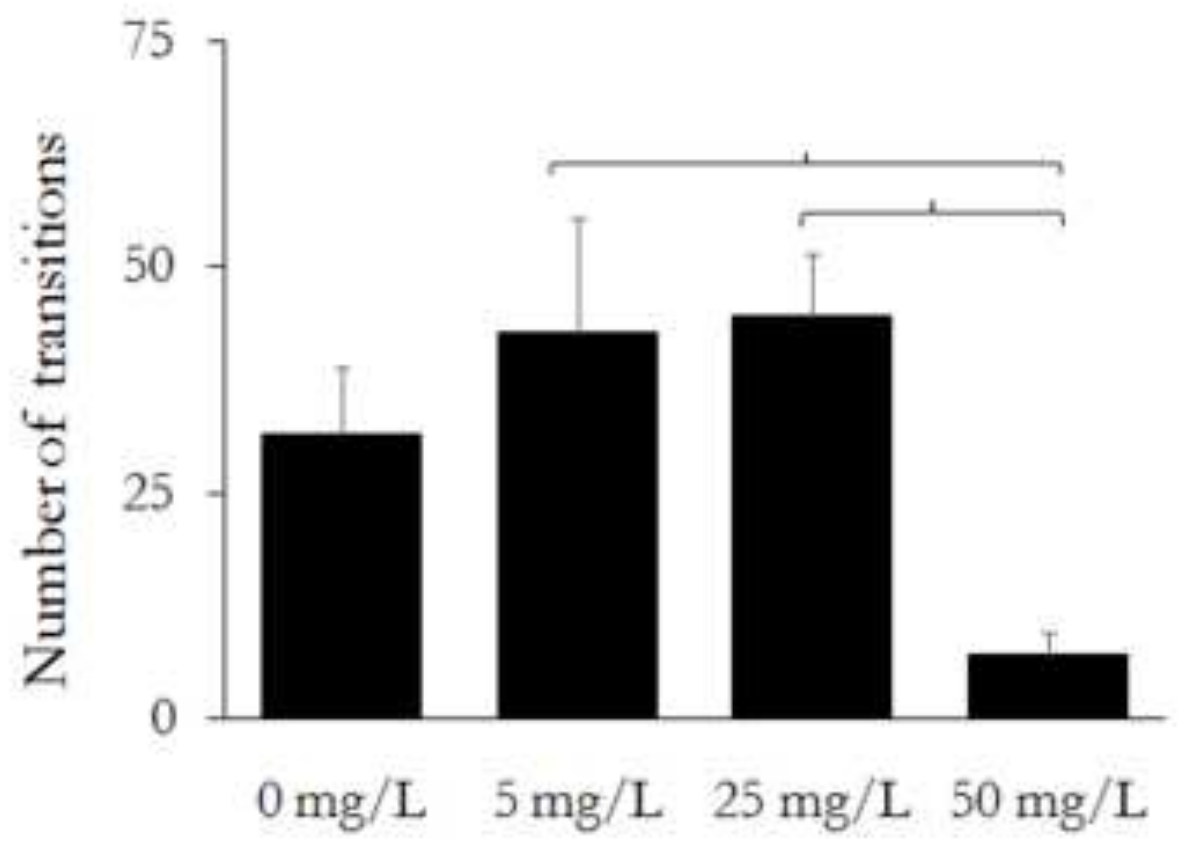




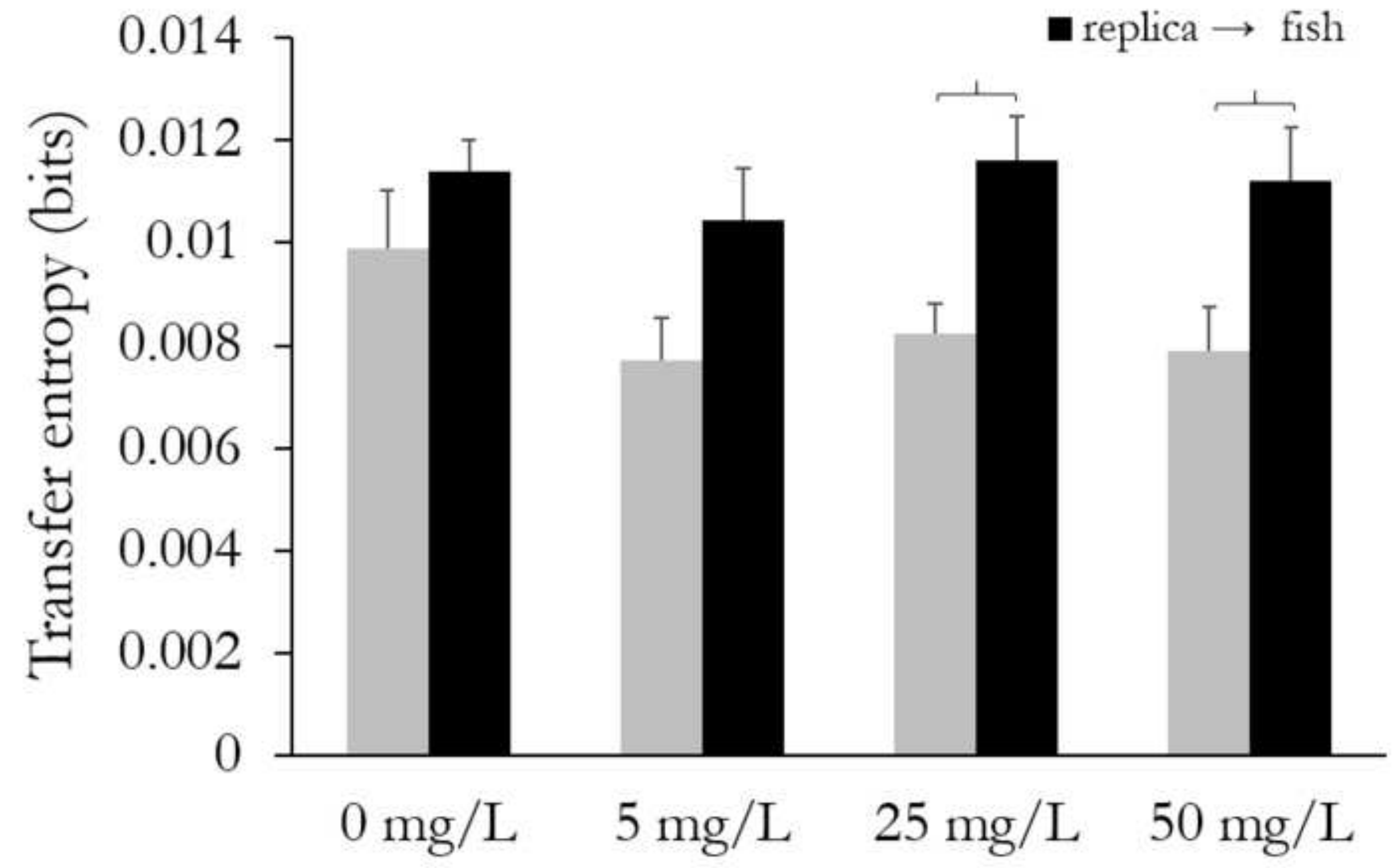

In fish $\rightarrow$ replica

- replica $\rightarrow$ fish 


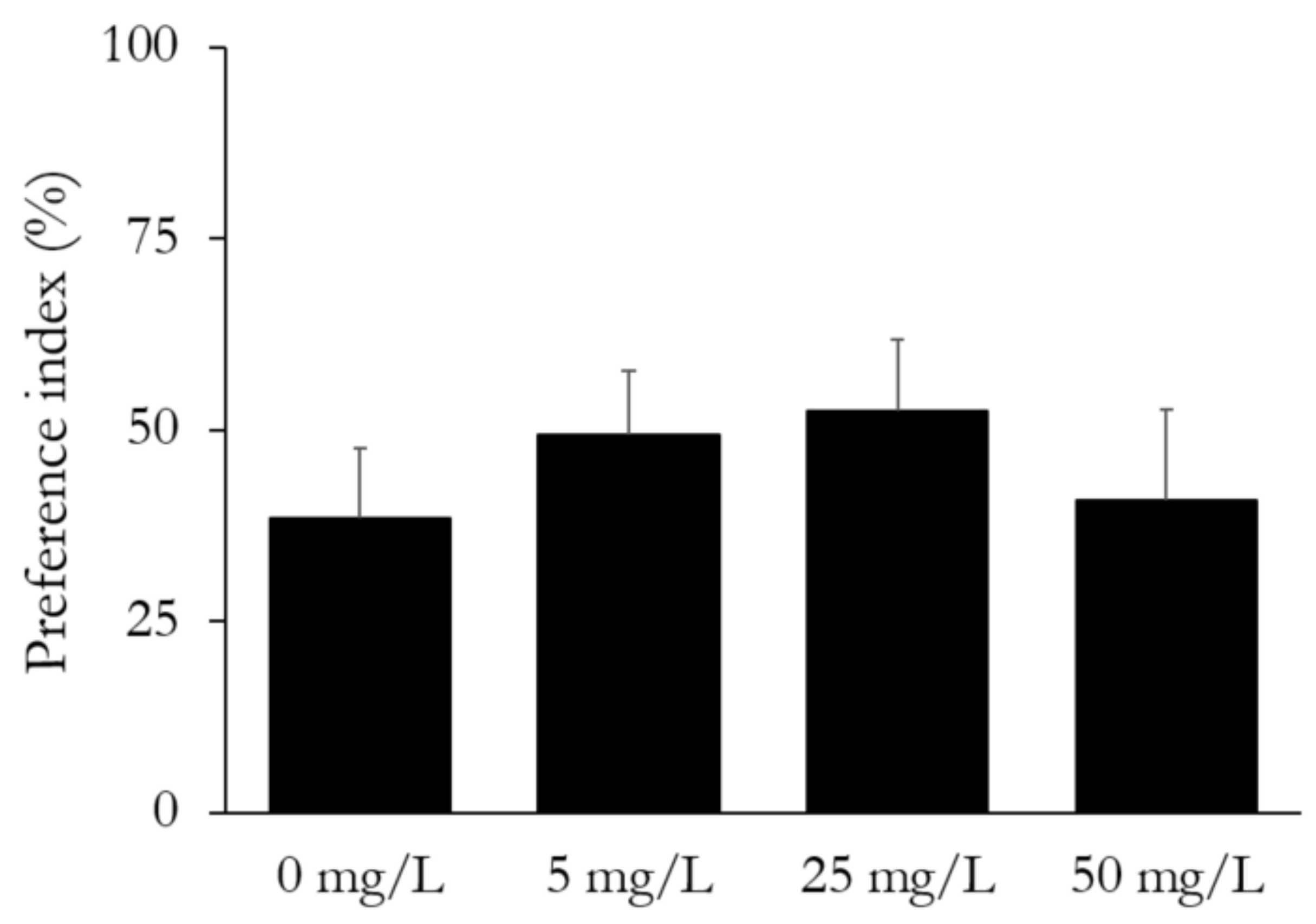

